\title{
Fair Cooperative Resource Allocation Schemes for Foggy Free Space Optical Network
}

\author{
Abdallah S. Ghazy ${ }^{1}$, Hossam A. I. Selmy ${ }^{2}$, Hossam M. H. Shalaby ${ }^{1}$ \\ ${ }^{1}$ Egypt-Japan University of Science and Technology (E-JUST), Alexandria 21934, Egypt \\ ${ }^{2}$ Cairo University, Cairo, Egypt \\ Abdallah.Ghazy@ejust.edu.eg, hossamselmy@yahoo.com,shalaby@ieee.org
}

\begin{abstract}
Two new resource allocation schemes are proposed for foggy reconfigurable free space optical (FSO) networks. The schemes are formulated as multi-objective optimization problems (MOP). The MOP is composed of lexicographic lex-max-min and Lex-min-max optimization. One of these schemes enhances reliability and fairness over network capacity while the other scheme does the opposite. The simulation results reveal that two schemes have higher reliability and fairness over robust static topology (relay network).
\end{abstract}

Keywords: Free space optical, Foggy weather, Resource allocation, Lex-Max-Min.

\section{Introduction}

With the significant development in the optical technology in the last decade, FSO links present promising and feasible solutions for the last mile connectivity problem. Currently, more FSO links are deployed in a given service area to meet the user's huge demands on Internet services and applications. However, the FSO channel is affected by various weather conditions. One of these conditions is the foggy weather, which absorbs and scatters transmitted optical signals [1]. The implemented network topology has to satisfy the required quality of service (QoS) at different visibility values.

A conventional FSO network implements static direct links between fiber backbone node and users as indicated in Fig. 1(A). Although, this static topology has a simple and low cost implementation, it has the worst communication performance against the limited visibility conditions. To overcome this performance degradation, the serial-relayed topology is addressed [1]. In this topology, one or more relays are inserted between far users and backbone node. The relay has two optical transceivers and is located at equal distances from other nodes as optimal placement [2], as indicated in Fig. 1(B). By increasing the number of intermediate relays between user and backbone node, the best FSO link performance could be achieved. Obviously, this enhancement in the network performance comes at a significant increase in the network cost. A more robust static FSO network is achieved by implementing mesh topology [3]. In this topology, redundant FSO links are used between users to keep connectivity to the backbone node as indicated in Fig. 1(C). Clearly, at a given atmospheric condition, each user selects the path that achieves the highest transmission rate at an acceptable bit-error rate. Generally, enhancing the performance of static FSO networks requires installation of a large number of redundant optical transceivers, which in turn raises the network cost.

Better performance could be achieved at a reasonable lower cost by implementing dynamic (reconfigurable) FSO network topologies [4]. These dynamic topologies are classified according to network resources sharing into cooperative and non-cooperative topologies. In dynamic noncooperative FSO network topologies no resources are shared among different users [4]. Users with bad links switch their traffic to users with relatively better links and the transmission rate of each user is kept the same. This is achieved by increasing transmission rates of good links to be sum of switched transmission rates. Clearly, increasing transmission rate of an optical link is not feasible and has practical limitations [5].

To overcome these limitations, dynamic cooperative topologies are introduced. In reconfigurable cooperative FSO network topologies, users with bad optical links switch their transmissions to users with better links and share their links capacities. Clearly, transmission rates of good optical links are divided among nodes and switched transmissions in order to keep quality of service for switched ones. In other words, the network users cooperate and share their resources (optical bandwidths) to keep connectivities between backbone node and users with bad links, i.e., increase FSO 


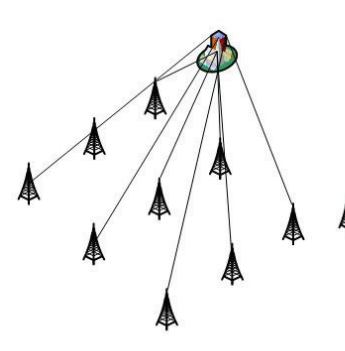

(A)

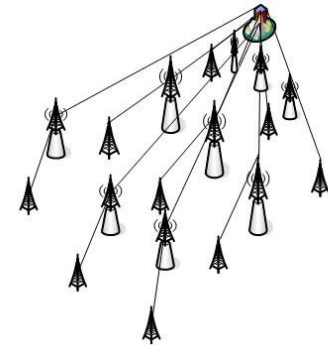

(B)

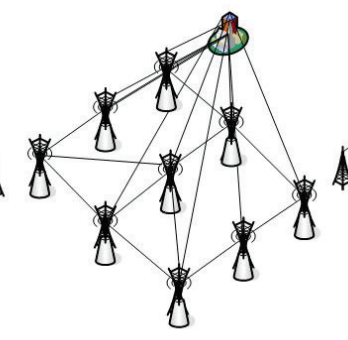

(C)

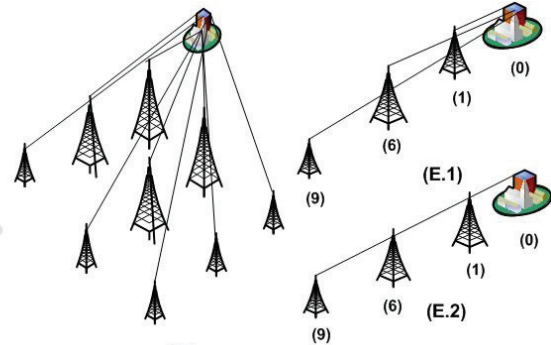

(D)

(E)

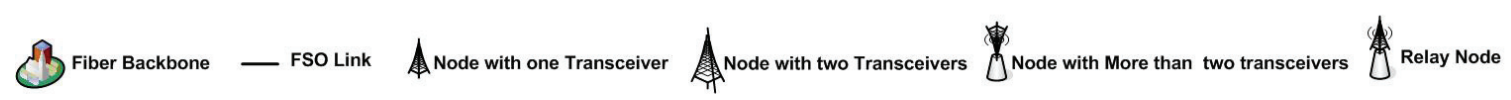

Fig. 1. Four FSO network models: nine nodes beside the backbone node; (A) Direct-Link 'D-L', (B) Relayed-Link 'R-L', (C) Mesh, and (D) Reconfiguration-Cooperation. (E) Network reconfiguration of 'Reconfiguration-Cooperation' model. Each network is $3 \times 3 \mathrm{~km}^{2}$ and each cell is $1 \times 1 \mathrm{~km}^{2}$.

network reliability (decrease number of dropped nodes) during foggy weather. Moreover, at given weather conditions, the network resources could be fairly allocated (achieves near the same transmission rate to backbone node) among different users by implementing proper resource allocation scheme. Also, here the number of optical transceivers available at each node is still playing an important role in the network performance. However, this number is much lower than that required in static topologies to achieve the same performance.

An example of reconfigurable cooperative FSO network that consists of a backbone node and nine nodes is indicated in Fig. 1(D). In this network, each node of the inner four nodes has two transceivers while each node of the outer five nodes has one transceiver. At clear weather, all nine nodes are connected to the backbone directly, as indicated in Fig. 1(E.1). But at the lower visibility, each node can switch to its neighbor node to maintain its connectivity to the backbone as in Fig. 1(E.2).

In this paper, two fair cooperative resource allocation schemes are proposed to enhance the reliability of dynamic cooperative FSO network (Fig.1(D)) in foggy weather conditions at low implementation cost. Also, the performance of proposed schemes is evaluated and compared to that of the relayed networks to measure the achieved performance improvement.

\section{FSO Link Model}

The FSO channel losses are accumulated from many sources, e.g., atmospheric variations (fog, rain, and snow), beam spreading (geometric), and scintillation, [6]. In the considered networks, both fog and geometrical losses are assumed to be the dominating ones. The considered fog loss is defined by several empirical models, the Kim model is used, which is the most accurate one for low visibility $(V \leq 6 \mathrm{~km}$ ) FSO link [7]. Moreover, a homogeneous foggy weather is assumed over the entire network with equal visibility value. The used geometric loss is described in [5]. Two sources contribute to the noise of FSO links, namely, shot and thermal noises. When the background radiation level is relatively high (outdoor), the thermal noise can be ignored and the receiver can be modeled as shot noise limited [8]. In addition, on-off Keying (OOK) modulation is considered for all FSO links.

\section{Reconfigurable Cooperative FSO Network Topology Parameters}

Generally, the cooperative FSO network consists of $N$ nodes $v=\left(v_{0}, v_{1}, \ldots, v_{N}\right)$ with arbitrary geographical distribution in addition to the backbone node $v_{0}$. The number of optical transceivers at $k$ th node is denoted by $Z_{k}, k \in\{1,2, \ldots, N\}$. In the considered FSO network, the inner $n_{2}$ nodes near to the backbone node are assumed to have two transceivers while the far $n_{1}=N-n_{2}$ have only one transceiver, $Z_{k} \in\{1,2\}$. Also, the backbone node is equipped with $N$ transceivers.

The losses of different FSO links (fog and geometric attenuations) are characterized by a $\gamma$ matrix, $\gamma=\left(\gamma_{00}, \ldots, \gamma_{0 N} ; \ldots, \gamma_{i j}, \ldots ; \gamma_{N 0}, \ldots, \gamma_{N N}\right)$, where $\gamma_{i j}$ is the loss coefficient of link between transmitter of $i$ th node and receiver of $j$ th node. Clearly, $0 \leq \gamma_{i j} \leq 1, \gamma_{i i}=0, \gamma_{i j}=\gamma_{j i}$ for any $\{i, j\} \in\{0,1, \ldots, N\}$. At a given foggy weather state, the cooperative FSO network could be connected with different fea- 
sible configurations (connection matrices) that achieve the minimum QoS parameters (bit rates at bit error rates which don not exceed the bit error rate threshold). The number of these matrices is $\Lambda$. For $l$ th configuration, $l \in\{1,2, \ldots, \Lambda\}$, the connection status between network nodes are summarized by connections matrix $G_{l}=\left(g_{l 00}, \ldots, g_{l 0 N}, \ldots, g_{l i j}, \ldots, g_{l N 0}, \ldots, g_{l N N}\right)$, where $g_{l i j}$ is the connection status between $i$ th and $j$ th nodes in configuration $l$ and $g_{l i j} \in\{0,1\}$. The connection between nodes $i$ and $j$ is established if $g_{l i j}=1$. Also, bidirectional links are assumed so that $g_{l i j}=g_{l j i}$ and $g_{l i i}=0$. Moreover, all FSO links are assumed to have the same average transmitted power, i.e., the power of optical link between nodes $i$ and $j$ in configuration $l$ is constant, $P_{l i j}=P$. However, to increase link capacity and guarantee an error rate less than a specified maximum $B E R_{l i j}<B E R_{\max }$, the connection between nodes $i$ and $j$ in configuration $l$ adapts its transmission rate, $T_{l i j}$, to be one of $m+1$ discrete values, where $T_{l i j} \in\left\{x_{1}, x_{2} \ldots, x_{m}, 0\right\}$ and $x_{1}>x_{2}>\ldots>x_{m}$.

The transmission rate of node $k$ in configuration $l$ is denoted by $T_{l k}$, where $T_{l k}=\sum_{j=0}^{N} T_{l k j}$. The data rate of node $k$ (its own traffic) through connection to node $j$ in configuration $l$ is denoted by $R_{l k j}$. The overall data rate of node $k$ in configuration $l$ is $R_{l k}=\sum_{j=0}^{N} R_{l k j}$. Obviously, $R_{l k} \leq T_{l k}$ and $\left\{R_{l k}, T_{l k}\right\} \in\left\{x_{1}, x_{2}, \ldots, x_{m}, 0\right\}$. The end-to-end bit error rate of node $k$ in configuration $l, B E R_{l k}$, is bounded by $B E R_{l k} \leq B E R_{\max }$. The bit rate and bit error rate for all nodes associated with all feasible configurations could be summarized in $(\Lambda \times N)$ matrices $R$ and $E$, respectively. For a given configuration $l$, the bit rate for all nodes is represented in vector $(1 \times N) r_{l}, r_{l} \in R$. Also, the bit error rates in that configuration is summarized in vector $(1 \times N) e_{l}, e_{l} \in E$. The network capacity associated with configuration $l$ is $C_{l}=\sum_{k=1}^{N} R_{l k}$, and all capacities associated with all feasible configuration could be summarized in vector $(\Lambda \times 1) C, C_{l} \in C$. Also, the maximum capacity is defined by $C_{\max }=$ $\sum_{k=1}^{N} T_{k 0}$. Clearly, $C_{l} \leq C_{\max }$ and capacity utilization is computed as $U_{l}=C_{l} / C_{\max }$. The size of the feasible space, $\Lambda$, can be defined as the upper bound by the following inequation:

$$
\Lambda<\left[2^{N+1}-1\right] \times\left[\sum_{i i=0}^{2}\left(\begin{array}{c}
N+1 \\
i i
\end{array}\right)\right]^{\left(n_{2}\right)} \times\left[\sum_{j j=0}^{1}\left(\begin{array}{c}
N+1 \\
j j
\end{array}\right)\right]^{\left(n_{1}\right)} .
$$

\section{Proposed Lex-Max-Min Fairness and Lex-Max-Min Constrained Fairness Schemes.}

Toward increasing reconfigurable FSO network reliability and improvement fairness between network nodes, two resource allocation schemes are proposed.

The first scheme, called Lex-Max-Min Fairness (LMMF), selects from the feasible $\Lambda$ configurations the ones that maximize the minimum bit rate for all nodes. For configurations that have the same max-minimum bit rate, the LMMF scheme proceeds to select from them the configurations that have next max-minimum bit rate (sequential max-min optimization) [9]. If there are more than one vector solution with the same sequential max-minimum values, the LMMF selects the configuration that has sequential minimum of maximum bit error rate values (sequential minmax optimization). This scheme is formulated as multiple objective optimization problem 'MOP'. There are several methods to treat this optimization problem [10]. Lexicographic method is used to optimize these conflicted objectives [10]. Lexicographic represents the problem in two levels of optimization based on the priorities between the objectives as in:

$$
\begin{aligned}
& \text { Lex-Max-Min }\left\{r_{l}=\left(R_{l 1}, R_{l 2}, \ldots, R_{l N}\right): r_{l} \in R\right\} . \\
& \text { Subject to }: B E R_{l k} \leq B E R_{\text {max }}, R_{l k} \in\left\{x_{1}, x_{2}, \ldots, x_{m}, 0\right\}, P_{l i j}=P, P_{l i i}=0, Z_{k} \in\{1,2\}, k \in\{1, \ldots, N\}, \\
& l \in\{1, \ldots, \Lambda\},\{i, j\} \in\{0,1, \ldots, N\} . \\
& \text { Lex-Min-Max }\left\{e_{l}=\left(B E R_{l 1}, B E R_{l 2}, \ldots, B E R_{l N}\right): e_{l} \in E\right\} .
\end{aligned}
$$

Several constraints are associated with first optimization level. The goal of this optimization is to increase both network reliability and fairness among different nodes.

The second proposed resource allocation scheme is called Lex-Max-Min Constrained Fairness (LMMCF). This scheme aims to increase network capacity then improve both network reliability and fairness among different nodes. Additional optimization level is added to optimization problem 
of LMMF as indicated in:

$$
\begin{aligned}
& \operatorname{Max}\left\{C_{l}=\sum_{k=1}^{k=N} R_{l k}: C_{l} \in C\right\} . \\
& \text { Subject to }: B E R_{l k} \leq B E R_{\max }, R_{l k} \in\left\{x_{1}, x_{2}, \ldots, x_{m}, 0\right\}, P_{l i j}=P, P_{l i i}=0, Z_{k} \in\{1,2\}, k \in\{1, \ldots, N\}, \\
& l \in\{1, \ldots, \Lambda\},\{i, j\} \in\{0,1, \ldots, N\} . \\
& \text { Lex-Max-Min }\left\{r_{l}=\left(R_{l 1}, R_{l 2}, \ldots, R_{l N}\right): r_{l} \in R\right\} . \\
& \text { Lex-Min-Max }\left\{e_{l}=\left(B E R_{l 1}, B E R_{l 2}, \ldots, B E R_{l N}\right): e_{l} \in E\right\} .
\end{aligned}
$$

Equations (2) and (3) could be solved using exhaustive search to obtain the optimal solution(s).

\section{Simulation and Numerical Results}

In this section, we present numerical evaluations for the three networks in Fig. 1(A), (B) and (D), in addition to the partial relayed network 'P-R-L' (one relay for each one of three remotest nodes). The number of the transceivers for previous networks are 18, 36, 22, and 24 respectively, as shown in Fig. 1. Table 1 shows the simulation parameters of the FSO links. The values are selected to be in the practical range. Also, Jain's index $0 \leq F \leq 1$ is used to measure the network fairness [11].

Table 1. Simulation Parameters

\begin{tabular}{|c|c|c|c|}
\hline Link parameters & Values & Link parameters & Values \\
\hline Signal wavelength & $1550 \mathrm{~nm}$ & Divergence angle & $2 \mathrm{mrad} / \mathrm{m}$ \\
\hline Average Transmitted Power & $-12 \mathrm{dBm}$ & Bit Rates in Gbps & $1,3 / 4,2 / 3,1 / 2,1 / 3,1 / 4,0$. \\
\hline Diameter of Transmitter & $4 \mathrm{~cm}$ & Average ambient noise & $-49.6 \mathrm{dBm}$ \\
\hline Diameter of Receiver & $20 \mathrm{~cm}$ & BER threshold & $10^{-4}$ \\
\hline
\end{tabular}

As indicated in Fig. 2(A), for all compared schemes, all nodes are dropped when $V \leq 200 \mathrm{~m}$ and none is dropped for $V \geq 2.8 \mathrm{~km}$. In the range $200 \mathrm{~m} \leq V \leq 2.8 \mathrm{~km}$, different schemes have different performances. Specifically, at $V=1.4 \mathrm{~km}$ the D-L, P-R-L, and MMF/MMCF drop 6, 3, and 2 nodes respectively. Although in this case, LMMF and LMMCF schemes have identical performance, but generally the reliability of LMMF is better than the LMMCF due to its flexibility in link reconfiguration (no capacity constraint).

Figure 2(B) shows the fairness, $F$, between 9 nodes in the network capacity. For all schemes, at $V \geq 3.4 \mathrm{~km}$, the $F$ achieves it maximum value $(F=1)$. For $V \leq 2 \mathrm{~km}$, LMMF and LMMCF improve $F$ and outperform P-R-L. Specifically at $V=1.8 \mathrm{~km}$, LMMF performs better than LMMFC and the values of $F$ are $0.98,0.85$, and 0.58 for R-L/LMMF, P-R-L/LMMCF, and D-L, respectively.

Figure 2(C) shows the capacity of the network for different compared schemes, $C_{l}$. At clear weather, i.e., $V \geq 3.8 \mathrm{~km}$, all schemes achieve the same network capacity (9 Gbps). Moreover, as expected, the capacity achieved by LMMF is lower than that of both LMMCF and D-L. Specifically, at $V=2.2 \mathrm{~km}$ the capacity achieved by D-L and LMMCF is 6 Gbps while the one achieved by LMMF is $5.5 \mathrm{Gbps}$. This rises from the maximization of the capacity optimization in LMMCF case.

Figure 2(D) explains bit error-rate performance for different schemes. The proposed schemes have lower performances (higher bit error rates) than that of R-L then P-R-L schemes. Clearly, the proposed schemes aim to increase the fairness over the bit error rates.

The optimal solution for the proposed schemes could be computed off line, then it could be registered as lookup table in FSO tracking controller, which reconfigures the topology to optimal configuration versus the visibility in real time environment.

\section{Conclusion}

Two resource allocation schemes, namely, MMF and MMCF have been proposed to increase both reliability and fairness of reconfigurable FSO networks. The proposed schemes outperform both D-L and P-R-L traditional schemes. In addition, MMF and MMCF have different optimization criteria, where MMF gives the priority for fairness and reliability over the capacity, while MMCF does the opposite. 


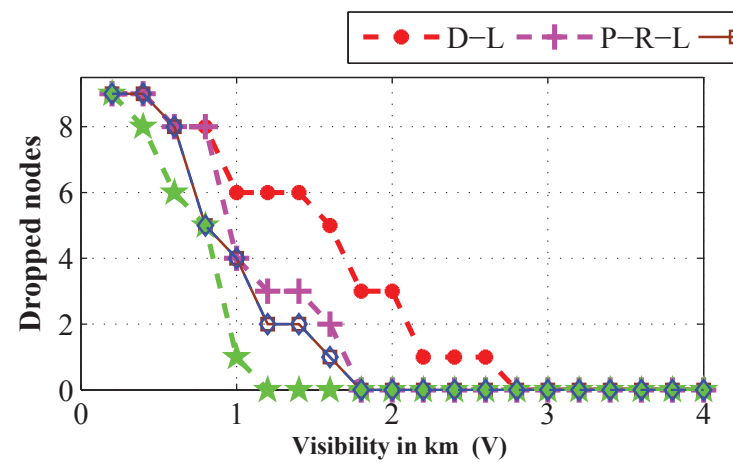

(A)

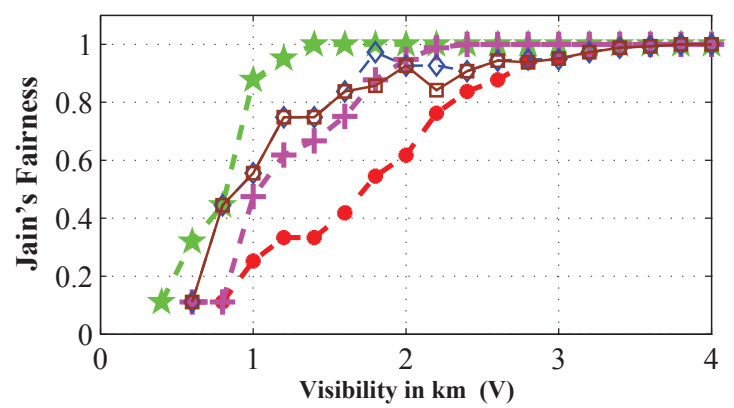

(B)

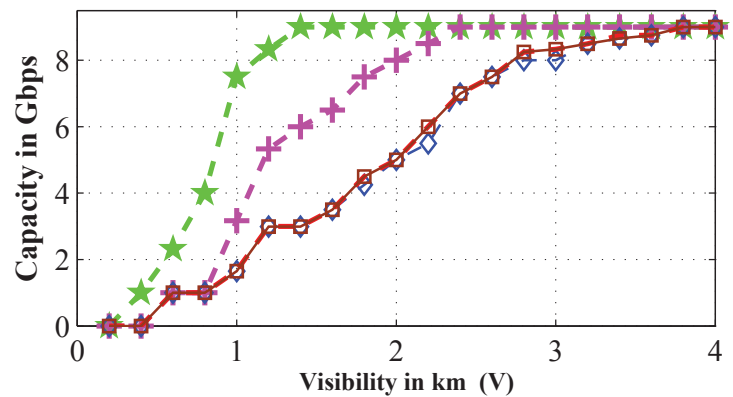

(C)

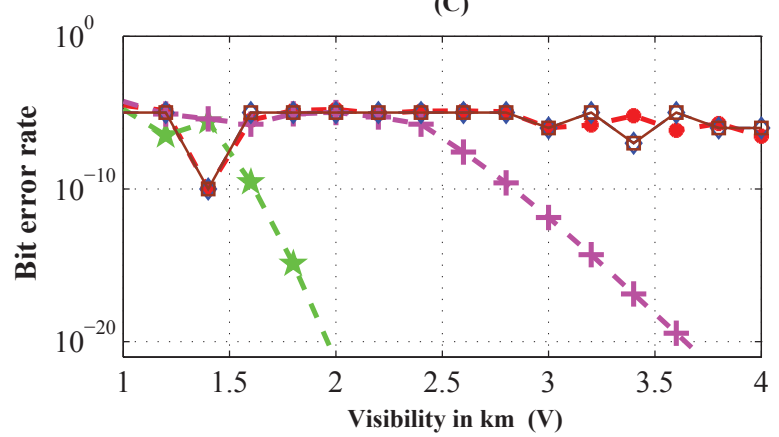

(D)

Fig. 2. Performance measures of five networks (A) Reliability, (B) Fairness, (C) Capacity, and (D) Error rate.

\section{Acknowledgement}

This work was funded by Science and Technology Development Fund (STDF-12670) and supported by the Ministry of Higher Education (MoHE), Egypt.

\section{References}

1. A. Vavoulas, H. G. Sandalidis, and D. Varoutas, "Weather effects on fso network connectivity," Journal of Optical Communications and Networking, vol. 4, no. 10, pp. 734-740, 2012.

2. M. A. Kashani, M. Safari, and M. Uysal, "Optimal relay placement and diversity analysis of relay-assisted free-space optical communication systems," Journal of Optical Communications and Networking, vol. 5, no. 1, pp. 37-47, 2013.

3. Z. Hu, P. Verma, J. Sluss Jr et al., "Improved reliability of free-space optical mesh networks through topology design," Journal of Optical Networking, vol. 7, no. 5, pp. 436-448, 2008.

4. S. D. Milner, T.-H. Ho, I. I. Smolyaninov, S. Trisno, and C. C. Davis, "Free-space optical wireless links with topology control," in International Symposium on Optical Science and Technology. International Society for Optics and Photonics, 2002, pp. 175-180.

5. S. Bloom, E. Korevaar, J. Schuster, and H. Willebrand, "Understanding the performance of freespace optics [invited]," Journal of optical Networking, vol. 2, no. 6, pp. 178-200, 2003.

6. I. Recommendation, "Prediction methods required for the design of terrestrial free-space optical links," International telecommunication Union, p. p.1814, 2007.

7. I. I. Kim, B. McArthur, and E. J. Korevaar, "Comparison of laser beam propagation at $785 \mathrm{~nm}$ and $1550 \mathrm{~nm}$ in fog and haze for optical wireless communications," in Information Technologies 2000, International Society for Optics and Photonics. IEEE, 2001, pp. 26-37.

8. R. M. Gagliardi and S. Karp, Optical Communications, 2nd ed. New York: Wiley, 1995.

9. W. Ogryczak and T. Sliwinski, "Lexicographic max-min optimization for efficient and fair bandwidth allocation," in International network optimization conference (INOC), 2007.

10. R. T. Marler and J. S. Arora, "Survey of multi-objective optimization methods for engineering," Structural and multidisciplinary optimization, vol. 26, no. 6, pp. 369-395, 2004.

11. R. Jain, D. Chiu, and W. Hawe, "A quantitative measure of fairness and discrimination for resource allocation in shared computer systems," arXiv preprint cs /9809099, 1998. 